\title{
PLASMA IMMERSION ION IMPLANTATION WITH LITHIUM ATOMS*
}

\author{
R. M. Oliveira ${ }^{\xi}$, M. Ueda, J. O. Rossi, B. Diaz \\ National Institute for Space Research, PO Box 515, ZIP 12245-970 \\ São José dos Campos, São Paulo, Brazil
}

\begin{abstract}
A new method was developed with the aim to creating plasma of lithium for plasma immersion ion implantation. First an argon glow discharge with operation pressure ranging from $2 \times 10^{-2}$ mbar to 1 mbar is generated by negatively polarizing an electrode from $-400 \mathrm{~V}$ to -1500 V. Small pieces of metallic lithium, $99.9 \%$ pure; fill the top of a conic crucible, with $2 \mathrm{~cm}$ depth, in electric contact with the electrode. Argon ions from the plasma are used to bombard this target where heat is created by the momentum transfer between the impacting ions and the crucible. By controlling the operation pressure and the electrode voltage polarization it is possible to easily heat the crucible to temperatures above the lithium melting point $\left(180^{\circ} \mathrm{C}\right)$, causing its evaporation. Lithium atoms are then ionized due to collisions with plasma particles. Double Langmuir probe measurements indicated variation on the density of the discharge from $4 \times 10^{9} \mathrm{~cm}^{-3}$ to $10^{10}$ $\mathrm{cm}^{-3}$, after lithium evaporation. Silicon wafer pieces immersed into this mixed plasma were submitted to repetitive negative high voltage pulses $(3 \mathrm{kV} / 6 \mu \mathrm{s} / 3 \mathrm{kHz})$, in order to attract plasma ions. High resolution X-Ray diffraction performed in these samples revealed the presence of high strain on the analyzed layers, in comparison with samples submitted just to the argon discharge.
\end{abstract}

\section{I.INTRODUCTION}

Several established processes have been successfully employed to treat the surface of different kind of materials, by making use of distinct physical principles. Classical examples are the Plasma Nitriding (PN) and the Plasma Immersion Ion Implantation (PIII). The first one is a thermally-driven and diffusion-based process which produces thick and hard case layers [1]. The other one is a non-thermal and ballistic process, bringing about relatively thin, albeit hard, case layers [2]. However, neither PN nor conventional PIII can perform treatments by using solid elements as needed for treatments with many metallic species. This is an important issue because metal-ion implantation has shown significant advantages over gaseous ion implantation in several applications [3].

\footnotetext{
$\xi_{\text {email: rogerio@plasma.inpe.br }}$

"Work supported by Brazilian Funding Agency (FAPESP)
}

In the case of PN, this drawback was mitigated by the introduction of double glow plasma surface alloying [4]. For PIII, metal implantation can be carried out by arc plasma devices but with the problem of macroparticles. Recently, a PIII mechanism using metal plasma electron evaporation source [5] was used to implant $\mathrm{Cr}$ and $\mathrm{Ti}$ ions in silicon wafers.

The process presented in this paper makes use of a hybrid discharge [6] running in high and low pressures, in which argon played the role of the carrier to heat a crucible containing the solid element (lithium). The pressure of the discharge is reduced after lithium plasma is established and the substrates are polarized by a high frequency pulser to draw ion species and to perform ion implantation and/or deposition.

Lithium was chosen initially due to its low melting/vaporization point and ionization potential of 5.39 $\mathrm{eV}$, but this method can be extended to perform PIII with many other chemical elements.

\section{EXPERIMENTAL}

The design of the experimental apparatus used to produce lithium plasma and further implantation of lithium ions at the surface of silicon samples is shown in Fig. 1.

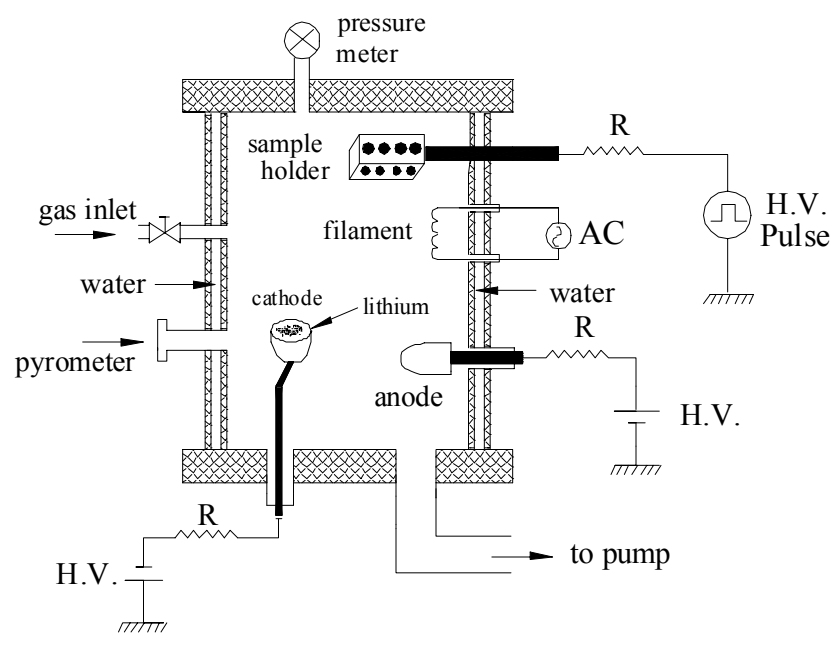

Figure 1. Experimental apparatus used to perform PIII with lithium atoms. 
To start the process, initially argon gas fills a waterrefrigerated stainless steel chamber, 601 volume, until a pressure of approximately 1 mbar is established. An argon glow discharge is turned on when the cathode, which consists of a conic molybdenum crucible $2 \mathrm{~cm}$ deep hosting small pieces of metallic lithium, is negatively polarized by DC voltages ranging from $400 \mathrm{~V}$ to $1 \mathrm{kV}$. A hollow cathode effect is observed, with the plasma being concentrated around the electrode. The high flux of argon ions reaching the cathode leads to a fast heating of the target. The temperature of the crucible reaches $400{ }^{\circ} \mathrm{C}$ easily, which is much higher than the melting point of the lithium $\left(180^{\circ} \mathrm{C}\right)$.

Once evaporated, lithium atoms are ionized due to collisions with plasma particles. The process is selfsustained and, as the lithium is being evaporated and ionized, more ion collisions happen, causing further increase of the temperature of the crucible and higher evaporation rate of lithium, as previously predicted $[7,8]$. If the pressure of the discharge and the polarization of the electrode remain constant, the temperature of the crucible begins to increase exponentially. In fact, temperatures as high as $800{ }^{\circ} \mathrm{C}$ were measured by an optical pyrometer viewing the crucible through a side glass window. Even though very high lithium evaporation rate occurs for this case, the pressure of the discharge is reduced in order to allow the plasma to spread out to fill the processing chamber steadily.

The pumping system which is composed of a two-stage rotary pump $\left(10 \mathrm{~m}^{3} / \mathrm{h}\right)$, a mechanical booster pump based on Roots principle $\left(375 \mathrm{~m}^{3} / \mathrm{h}\right)$, a diffusion pump and gate valves, which are manually switched, allows that the operation pressure remains in the range of $6 \times 10^{-3} \mathrm{mbar}$ to $1-3$ mbar, for the whole process.

When the operation pressure is decreased to $8 \times 10^{-3}$ mbar, the other electrode (anode) is positively polarized to further ionize the metal vapor. For this case, repetitive high voltage pulses $(3 \mathrm{kV} / 6 \mu \mathrm{s} / 3 \mathrm{kHz})$ as shown in Fig. 2, supplied by a Compact High Voltage Pulser $[9,10]$, are applied to the Si samples, causing lithium base coating and implantation.

The tungsten filament is important to control the plasma potential to avoid sputtering on the sample surface when lithium is not being implanted. Also, it is used as an electron source to start the argon glow discharge, switched on previously to the treatment for cleaning purposes.

A double Langmuir probe was used to measure the plasma density before and after lithium evaporation. Silicon test samples were taken to high resolution x-ray diffraction analysis. The X-ray diffraction measurements were carried out in a Phylips X'Pert diffractometer equipped with a four-crystal $\mathrm{Ge}(220)$ monochromator in the primary optics (between the $\mathrm{Cu} \mathrm{X}$-ray tube and the sample). In this configuration, the incident X-ray beam has an axial divergence of 12 arcsec and a wavelength dispersion of approximately $2 \times 10^{-4}$. An open detector in the secondary optics detected the diffracted beam. W-
Scans around the Si-(004) diffraction peak with the detector fixed at the $2 \Theta$ Bragg angle (rocking curve) were performed on the $\mathrm{Si}$ wafers before and after lithium implantation.

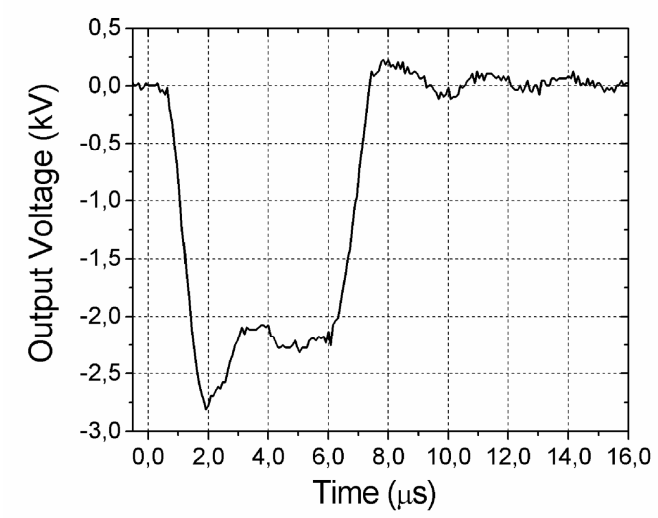

Figure 2. Pulse wavelenght applied to the sample during PIII treatment.

\section{RESULTS AND DISCUSSION}

Several experiments were performed to ensure the lithium evaporation and the production of lithium ions. An evidence of lithium evaporation was the change on the color of the discharge from violet (argon) to an intense red glow (lithium), besides from a sudden variation of the pressure of the discharge without any external control of the gas inlet valves. The degree of ionization varied significantly after lithium vaporization, since plasma density measured by double Langmuir probe varied from a maximum of $4 \times 10^{9} \mathrm{~cm}^{-3}$ to $1 \times 10^{10} \mathrm{~cm}^{-3}$. Fig. 3 shows experimental points collected by the electrostatic probe after lithium evaporation, while Fig. 4 shows the density values for an operation pressure of $2 \times 10^{-2} \mathrm{mbar}$, before and after lithium evaporation.

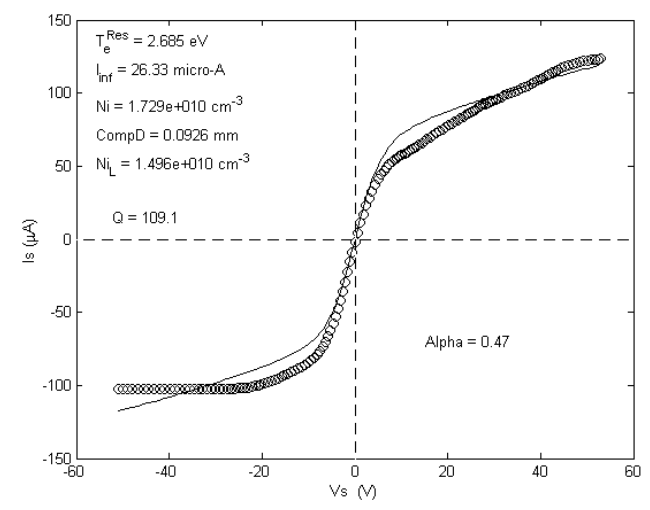

Figure 3. Double Langmuir probe measurements for the lithium plasma shown above: experimental points (circle) $\&$ theoretical fitting curve (solid).

Accordingly, plasma resistance fell down from $1.4 \mathrm{k} \Omega$ to $470 \Omega$, reflecting the change of $\mathrm{I} \times \mathrm{V}$ characteristic curve 
of the discharge. Indeed, a threefold increase of the current of the discharge was observed for a constant polarization voltage applied to the electrode, after lithium evaporation. In order to confirm the presence of lithium ions, a secondary electron detector was placed behind a negatively polarized metal grid $(-50 \mathrm{~V})$. In a comparison to the case of the argon glow discharge without lithium evaporation, the detected current density showed a sharp increase afterwards.

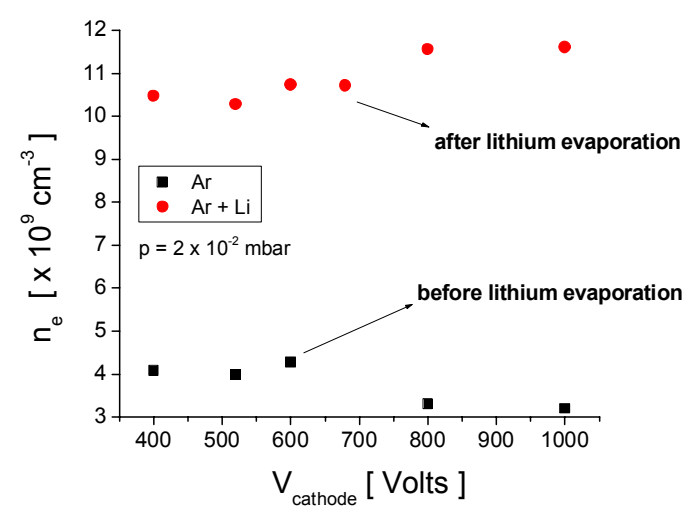

Figure 4. Plasma density for argon (square) and lithium (circle) glow discharges.

Even though a large amount of lithium ions had been detected in the plasma after lithium vaporization, it remained necessary to verify the effect of lithium ion implantation on $\mathrm{Si}$ test samples. This was accomplished by high resolution X-ray measurements, performed in silicon samples immersed into this plasma at an operation pressure of $8 \times 10^{-2} \mathrm{mbar}$ and submitted to pulses of $3 \mathrm{kV} /$ $2.5 \mathrm{kHz} / 5 \mu \mathrm{s}$. For this case, the rocking curve as shown in Fig. 5 indicates clear distortion for treated samples during 30 and 40 minutes in comparison to the symmetrical curve for the sample submitted just to the argon plasma. Such distortions were previously measured and interpreted as an effect of ion implantation in PIII treatments [11-13].

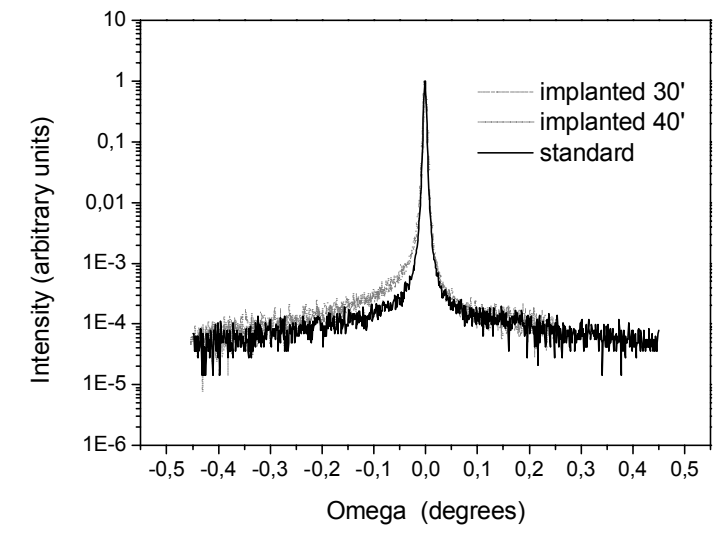

Figure 5. High resolution X-ray diffraction measurements for Si samples before and after PIII treatment with lithium atoms.

\section{SUMMARY}

Solid lithium was evaporated in an argon glow discharge in a process similar to a hollow cathode discharge seen in ion nitriding. Secondary electron detector and double Langmuir probe were used to diagnose the presence of lithium ions. Plasma density varied from $4 \times 10^{9} \mathrm{~cm}^{-3}$ to $10^{10} \mathrm{~cm}^{-3}$ after lithium evaporation. Plasma immersion ion implantation with lithium ions was carried out in test silicon samples, using a compact high voltage pulser. High-resolution X-ray diffraction analysis showed that lithium was successfully implanted.

\section{REFERENCES}

[1] A.H. Deutchman, R. J. Partyka and C. Lewis, Ind. Heat. 57 (1990), p. 32.

[2] J. R. Conrad, J. L. Radtke, R. A. Dodd, F. J. Worzala and N. C. Tran, J. Appl. Phys. 2 (1987), p.4951.

[3] A. Öztarhan, I. Brown, C. Bakkaloglu, G. Watt, P. Evans, E. Oks, A. Nikolaev and Z. Tek, Surf.\& Coat. Tech. 196 1-3 (2005), p. 327.

[4] Z. Xu, X. Liu, P. Zhang, Y. Zhang, G. Zhang and Z. He, Surf.\& Coat. Tech. 201 (2007), p. 4822.

[5] R. Wei, T. Booker, C. Rincon and J. Arps, Surf. Coat. Tech. 200 1-4 (2005), p. 579.

[6] R..M. Oliveira, M. Ueda, I. H. Tan, L. Hoshida and C. B. Mello, Plasma Processes and Polymers 4 (2007), p. S655.

[7] R.P. Doerner, M. J. Baldwin, R. W. Conn et al.. J. Nucl. Mater. 290-293 (2001), p. 166.

[8] R.P. Doerner, M. J. Baldwin, S. I. Krasheninnikov and D. G. Whyte, J. Nucl. Mater. 313-316 (2003), p. 383.

[9] J.O. Rossi, J.J. Barroso, M. Ueda and G. Silva, IEEE Transactions on Plasma Science, 34 (2006), p. 1757.

[10] J.O. Rossi, M. Ueda and J. J. Barroso, Brazilian Journal of Physics, 34 4B (2004), p. 1565.

[11] D.L. Chapek, J.R. Conrad, R.J. Matyl and S. B. Felch, J. Vac. Sci. Technol. B 12 (1994), p. 951.

[12] E. Abramof, A.F. Beloto, M. Ueda, G. F. Gomes, L. A. Berni and H. Reuther, Nucl. Instr. And Meth. in Phys. Res. B 161-163 (2000), p. 1054.

[13] B. Diaz, E. Abramof, R. M. Castro, M. Ueda and H. Reuther, Journal of Applied Physics 101, (2007), 103523. 